\title{
Prozessmanagement als Gestaltungshebel der digitalen Transformation?
}

Elke Brucker-Kley, Thomas Keller und Denisa Kykalová

1.1 Forschungsgegenstand und Begriffsbestimmung - 4

1.2 Forschungsfragen und Studiendesign - 7

1.3 Das Rahmenwerk der Studie - 11

Literatur - 16 
Bietet das Hype-Thema «digitale Transformation» die Chance, Prozessmanagement in ein ganz neues Licht zu rücken? Ist Prozessmanagement vielleicht sogar eine wesentliche Voraussetzung, um den digitalen Wandel zielgenauer zu gestalten? Oder wird Prozessmanagement im Zeitalter der selbstorganisierenden Teams und Customer Journeys letztlich überflüssig? Nicht nur Branchen, sondern auch Managementdisziplinen sind herausgefordert, sich mit der Relevanz der digitalen Transformation zu beschäftigen, sich neu zu ordnen, sich besser zu verzahnen und sich letztlich zu wandeln. Ziel der Business-Process-Management-Studie 2016, mit der das Institut für Wirtschaftsinformatik der Zürcher Hochschule für Angewandte Wissenschaften, School of Management and Law seit 2011 regelmässig Status quo und Best Practices im deutschsprachigen Raum erhebt, war es, die Rolle des Prozessmanagements als Gestaltungshebel der digitalen Transformation zu beleuchten.

\subsection{Forschungsgegenstand und Begriffsbestimmung}

I) Nach und nach wird jede Branche und jeder Lebensbereich von der Digitalisierung erfasst werden - von Produktion bis Dienstleistung, von Bildung bis Gesundheit. Der technische Fortschritt verheisst ein besseres, angenehmeres und längeres Leben und bedroht zugleich praktisch jedes etablierte Geschäftsmodell (GDI: Die Zukunft der vernetzten Gesellschaft (Frick und Höchli 2014)).

Die Business-Process-Management (BPM)-Studie 2016 widmet sich einem Thema, das für viele bereits zum Reizwort geworden ist. Das Schlagwort «digitale Transformation» dominiert nicht nur die Veranstaltungskalender, sondern in zunehmendem Masse die strategischen Diskussionen in Unternehmen aller Grössen und Branchen. Im Rampenlicht stehen mobile Apps oder vielversprechende digitale Helfer und technologische Gadgets, wie Serviceroboter oder Augmented-Reality-Brillen, die Kunden und Mitarbeitenden neue Dimensionen der Interaktion eröffnen sollen. Die Faszination ist gross, doch für Unternehmen ist häufig unklar, welche Relevanz diese Innovationen für ihr Geschäftsmodell haben und wie sie sie in die bestehenden Prozess- und Systemlandschaften integrieren können. Auch die allgegenwärtigen Warnungen, dass ein Moment der Unachtsamkeit genüge, um von einem innovativen Startup aus dem Markt gedrängt zu werden, tragen nicht gerade dazu bei, sich freudig dem Thema «Digitalisierung» zuzuwenden.

\section{- Digitale Transformation - Für wen?}

Dabei ist häufig unklar, was sich konkret hinter dem Begriff «digitale Transformation» verbirgt. Dass sich Unternehmen verändern und kundenzentriert sein sollen, ist nichts Neues. Auch Digitalisierung ist mehr als vier Jahrzehnte nach Erfindung des Mikroprozessors und mehr als zwei Jahrzehnte nach der Geburt des World Wide Webs keine neues Phänomen. Neu zu sein scheint die Geschwindigkeit, die diese Transformation aufgenommen hat und die Reichweite, mit der sie in alle Lebensbereiche eindringt und die Grenzen zwischen Mensch, Maschine und Unternehmen zunehmend aufweicht. Diese Tendenz wird nicht erst seit kurzem beobachtet. Die Suche nach einer Definition der digitalen Transformation zeigt, dass der Begriff bereits zur Jahrtausendwende in einem sehr ähnlichen Licht diskutiert wurde. So definieren (Stolterman und Fors 2004) in ihrem Research Position Paper «Technology and the Good Life» digitale Transformation als die Summe aller Veränderungen, die digitale Technologien in allen Aspekten des menschlichen Lebens verursachen (- Abb. 1.1). Sie leiten daraus die Forderung an die Informatikforschung ab, das eindimensionale Verständnis von Informationstechnologie 
um die Perspektive des menschlichen Erlebens zu erweitern. Technologien werden unter diesem Blickwinkel nicht nach rein funktionalen Kriterien für Nutzer in bestimmten Rollen (z. B. Kunde, Entscheider) entworfen, sondern für Menschen, die ein Leben führen und Bedürfnisse haben, die sich nicht ausschliesslich auf eine Lebenswelt begrenzen lassen, sondern kontextübergreifend sind. Sie reihen sich damit in eine Strömung des Information Systems Engineering ein, die seit Ende der 1990er-Jahre mit Disziplinen wie «Human-Centered Computing», «User Experience» oder «Interactions Design» das Anwendererlebnis in den Mittelpunkt des Systementwurfs stellt. Im selben Zeitraum entsteht der Begriff der «Customer Experience», erstmals erwähnt von (Pine und Gilmore 1998) in einem Artikel im Harvard Business Review, die mit einer sehr ähnlichen Haltung das positive Kundenerlebnis als wesentliche Basis einer nachhaltigen und für alle Seiten wertstiftenden Kundenbeziehung einstufen. Die sich in der Folge entwickelnde Disziplin des «Customer Experience Management» ist jedoch grundsätzlich technologieunabhängig und bezieht sich auf jeden Berührungspunkt mit dem Kunden, egal ob physisch oder digital. Wesentlich dabei ist die Anerkennung des Kundennutzens als relativen Wert aus Kundensicht («perceived value»). Beim wahrgenommenen Kundennutzen werden Preis und Qualität zu subjektiven Grössen, die von jedem Kunden individuell für jede Situation und abhängig von Erfahrungen, Vergleichen und Werthaltungen definiert werden (McDougall und Levesque 2000).

I) As goods and services become commoditized, the customer experiences that companies create will matter most ... A Rolling Stones concert-goer, for example, will pay a premium for an official T-shirt emblazoned with the date and city of the concert. That's because the price points are a function less of the cost of goods than of the value the buyer attaches to remembering the experience (Pine und Gilmore 1998).

\section{- Digitale Transformation - Wozu und wie?}

Doch welche Möglichkeiten haben Unternehmen, diese Kundenerlebnisse bewusst zu gestalten und ihre Produkte und Dienstleistungen auf Kundenbedürfnisse auszurichten, die sich kaum einschätzen lassen? Obwohl Pine und Gilmore (1998) die Wichtigkeit von Gerüchen und haptischen Erlebnissen betonen, sehen sie das Potenzial neuer Technologien. So beschreiben sie das Visionarium Reality Center des Grafikworkstation-Herstellers Silicon Graphics, das bereits 1996 dreidimensionale Produktvisualisierungen ermöglichte, mit denen Produktentwickler und Kunden verschiedene Produktvarianten mit allen Sinnen erproben konnten, um die vielversprechendsten frühzeitig zu erkennen. Seither ist viel geschehen. 3D-VirtualReality-Brillen für das ultimative Gaming- und Filmerlebnis sind heute für jedermann erschwinglich. Mobile Technologien, Social Media und Wearables gehören zum digitalen Alltag. Konsumenten verfügen über digitale Möglichkeiten und Kompetenzen, die Unternehmen zunehmend ausnutzen, um Interaktionen und Transaktionen unmittelbarer und schneller zu gestalten. Dieser Einsatz neuer Technologien steht folglich auch im Mittelpunkt vieler Definitionen und Analysen zum Thema «digitale Transformation». Auch die oft zitierte Definition der MIT Sloan Management Review, die im Rahmen einer Studie mit Cap Gemini entstand, stellt digitale Transformation mit der Anwendung neuer Technologien (Social Media, Mobile etc.) gleich (Fitzgerald et al. 2014, S. 2; - Abb. 1.1). Der Blick auf den Anwendungszweck dieser Technologien ergibt jedoch ein deutlich differenzierteres Bild. Mit den drei Bereichen «Customer Experience», «Operational Improvements» und «Business Model Change» wird der Rahmen für die Gestaltung der digitalen Transformation gesteckt. Innerhalb der operationalen Verbesserungen finden sich «alte Bekannte» wie Prozessdigitalisierung, Worker Enablement und Performance Management, die nicht perfekt in das Bild der neuen Technologien pas- 


\section{Für wen?}

«The digital transformation can be understood as the changes that the digital technology causes or influences in all aspects of human life. This research challenge has to be accepted on behalf of humans, not in their roles as users, customers, leaders, or any other role, but as humans living a life.»

(Stolterman \& Fors, 2004)

\section{Wozu?}

«We define Digital Transformation as the use of new digital technologies (social media, mobile, analytics or embedded devices) to enable major business improvements (such as enhancing customer experience, streamlining operations or creating new business models).»

(MIT Sloan Management Review Report, 2014)

\section{Wie? Womit?}

«lt's tempting to look for simple definitions, but to be meaningful and sustainable, we believe that digital should be seen less as a thing and more a way of doing things. To help make this definition more concrete, we've broken it down into three attributes: creating value at the new frontiers of the business world, creating value in the processes that execute a vision of customer experiences, and building foundational capabilities that support the entire structure.»

(McKinsey, 2015)

- Abb. 1.1 Definitionen für digitale Transformation

sen, aber dennoch von Unternehmen als wesentliches Element der digitalen Transformation betrachtet werden. Digitale Transformation scheint also nicht nur im Front-End, in der Interaktion mit Kunden stattzufinden, sondern beeinflusst auch die Gestaltung und Ausführung der operativen Prozesse. Ist dies der naheliegende Ansatzpunkt für das betriebliche Prozessmanagement? Oder greift diese Betrachtung, die das Prozessmanagement im Back-End-Silo der operativen Verbesserungen einordnet, zu kurz? Hat das Prozessmanagement auch Einfluss auf die Gestaltung der Kundenerlebnisse oder sogar das Potenzial, das Geschäftsmodell zu verändern und Produkt- und Serviceinnovationen zu schaffen? Könnte Prozessmanagement, sowohl im fachlich-strategischen als auch im technisch-operativen Sinn, eine der grundlegenden Fähigkeiten («foundational capabilities») sein, die gemäss (McKinsey und Co 2015) in Unternehmen vorhanden sein müssen, um ihr Geschäft wertstiftend digital transformieren zu können (- Abb. 1.1)?

An diesem Punkt setzt die BPM-Studie 2016 an und stellt sich folgende Frage: «Welche Rolle spielt Prozessmanagement als Gestaltungselement der digitalen Transformation?» Sie widmet 
sich den Themenschnittstellen der drei Wirkungsfelder «Kundenutzen - Digitalisierung - Prozessmanagement» und untersucht im Rahmen einer qualitativen und quantitativen Studie: - Den Status quo in der Praxis: In welcher Ausprägung setzten Unternehmen im deutschsprachigen Raum Konzepte, Methoden und Technologien an diesen Schnittstellen ein?

- Erfolgsmuster in Unternehmen: Welche Erfahrungen, Lösungsansätze und Resultate weisen Unternehmen auf, die den Kundennutzen und die Möglichkeiten der Digitalisierung ins Zentrum ihrer BPM-Initiativen stellen?

\subsection{Forschungsfragen und Studiendesign}

«Welche Rolle spielt das Prozessmanagement als Gestaltungshebel der digitalen Transformation?» lautet die Forschungsfrage der BPM-Studie 2016. Mögliche Chancen und Potenziale der Digitalisierung für das Prozessmanagement wurden in Forschungsfragen und Hypothesen formuliert. Im Rahmen einer quantitativen Online-Befragung wurde der Status quo in Unternehmen erhoben. Gemeinsam mit Unternehmen wurden Fallstudien erarbeitet und Erfolgsmuster abgeleitet.

Die Ergebnisse der BPM-Studie 2015 haben gezeigt, dass Unternehmen Prozessmanagement zunehmend mit dem Ziel einer stärkeren Kundenorientierung einsetzen (Brucker-Kley et al. 2015). Rund die Hälfte der befragten Unternehmen gab die Erhöhung der Kundenzufriedenheit als Motivation für das betriebliche Prozessmanagement an. Effizienz war in der Studie 2015 mit 61 \% die dominierende Zielsetzung für das Prozessmanagement, jedoch wurde Kundenzufriedenheit auf Platz zwei sogar häufiger genannt als das Ziel der Transparenz (38\%). Die Frage nach der Umsetzung ergab jedoch ein ernüchterndes Bild. Durchgängige und am Kundenbedarf ausgerichtete Prozesse standen nur für rund ein Viertel der befragten Unternehmen im Fokus. Fast 40 \% setzten keine Methoden oder Technologien ein, um ihre Prozesse flexibel an den Kontext oder die Bedürfnisse ihrer Kunden anzupassen und wenn, dann nutzten sie vordefinierte Geschäftsregeln, um komplexe stark strukturierte Prozesse zu steuern. Innovation war nur für rund $16 \%$ der befragten Unternehmen ein Thema für das Prozessmanagement. Lediglich $22 \%$ der befragten Unternehmen erhoben systematisch Automatisierungspotenzial. Ausgehend von den Ergebnissen der Studie des Vorjahres stellt sich die Frage, ob das Prozessmanagement in Unternehmen strategisch und operativ für die Herausforderungen des digitalen Wandels gerüstet ist. Kann das Prozessmanagement über Effizienzsteigerung und Automatisierung standardisierter Back-End-Prozesse hinaus einen Beitrag zu veränderten Kundenerlebnissen und innovativeren Produkten und Dienstleistungen leisten?

\section{- Effizienz versus Kundenerlebnis?}

Auch die Ergebnisse anderer jüngerer Studien zur Ausprägung des Prozessmanagements deuten darauf hin, dass es noch Entwicklungspotenziale in den für die digitale Transformation essentiellen Bereichen Kundenorientierung und Digitalisierung gibt. So resümiert der 2016 aktualisierte Digital Maturity \& Transformation Report (Universität St. Gallen und Crosswalk 2016), der auf einer Befragung von mehr als 500 Schweizer Unternehmen beruht, dass es insbesondere in den Bereichen Customer Experience und Prozessdigitalisierung niedrige Erfüllungsraten und einen hohen Aufholbedarf gäbe. Überprüft wurden beim Kriterium Prozessdigitalisierung unter anderen Geschäftsfähigkeiten wie Prozessautomatisierung, die Entscheidungsunterstützung durch Big-Data-Analysen und die Planung und die Durchführung von digitalen Marketingmassnahmen. Eine Studie von BPM\&O und BearingPoint (2015) bei 270 Unternehmen im deutschsprachigen Raum kam zum Schluss, dass das Prozessmanagement 
im Vergleich zu den Back-End-Operations und Supportprozessen (z. B. IT, Finanzen) in den Bereichen mit Kundenkontakt wie Marketing, Sales und Service vergleichsweise schwach ausgeprägt ist. Auch der Einfluss von Ergebnissen der Kundenzufriedenheitsmessungen auf die Prozessgestaltung ist laut dieser Studie noch begrenzt. Dass Unternehmen aber auch beim Thema Digitalisierung primär an stark strukturierte back-end-lastige Prozesse denken, erstaunt und bestätigt zugleich, dass Effizienzsteigerung auch im Zeitalter der digitalen Transformation eine Notwendigkeit bleibt. Diesen Schluss legt eine Studie von (Crisp Research 2015) mit über 500 Führungskräften in Deutschland nahe. Sie zeigt, dass $42 \%$ der befragten Unternehmen den grössten Einfluss der digitalen Transformation in der Optimierung von Produktions- und Logistikprozessen sehen, wohingegen die Digitalisierung der Kundenbeziehung mit $31 \%$ auf Platz zwei landet. Dieser Balanceakt zwischen Kundenperspektive und internem Fokus, zwischen technologischen Möglichkeiten und finanzieller Machbarkeit stellt Unternehmen unabhängig von Grösse und Branche vor grosse Herausforderungen.

\section{- Prozessmanagement - Kundenutzen - Digitalisierung}

Das spürt auch das traditionell auf Transparenz und Effizienzgewinne ausgerichtete Prozessmanagement, das sich vermehrt der Frage stellen muss, welche Rolle die durch den technologischen Wandel veränderte Kundenbeziehung beim Entwurf, bei der Operationalisierung und bei der Optimierung von Prozessen spielt. Wie lassen sich die neuen Möglichkeiten, die sich aus neuen Interaktionsformen mit Kunden und Mitarbeitenden ergeben, mit den vorhandenen oder zu schaffenden Fähigkeiten im Back-End in Einklang zu bringen? Wie lassen sich dabei sowohl durchgängige und effiziente Prozesse als auch überzeugende digitale und physische Kundenerlebnisse realisieren? Diese hohen Anforderungen an den Themenschnittstellen zwischen Prozessmanagement, Kundennutzen und Digitalisierung wurden im Rahmen der vorliegenden Studie als Forschungsfragen formuliert (- Abb. 1.2), mit den nachfolgenden Hypothesen hinterlegt und in den quantitativen und qualitativen Elementen der Studie adressiert.

\subsubsection{Hypothesen und Forschungsfragen}

\section{- Strategische Ausrichtung - Kundenzentrisches und technologienahes BPM}

Unternehmen nennen die verstärkte Kundenorientierung neben Effizienz und Transparenz als wichtige Motivation für das Prozessmanagement (Brucker-Kley et al. 2015). Dennoch spielt der Kundennutzen für Rechtfertigung und konkrete Ausgestaltung des Prozessmanagements eine untergeordnete Rolle. Prozessdesign, -automatisierung und -optimierung in Unternehmen sind primär auf Effizienz ausgerichtet. Auch die Business Cases für BPM-Investitionen (organisatorisch und technisch) basieren in der Regel auf Effizienzgewinnen. Kundenzufriedenheit bleibt ein strategisch formuliertes Ziel, das indirekt über Qualität, Geschwindigkeit und Preis unterstützt wird, das jedoch noch kaum mit unmittelbar wirksamen Massnahmen des operativen Prozessmanagements - wie etwa individualisierten Prozessen - adressiert wird.

\section{- Interne Sicht versus Kundenperspektive}

Digitale Transformation wird von Kunden und Mitarbeitenden getrieben, die die Potenziale moderner Technologien kennen und selbstverständlich erwarten, dass Unternehmen diese ausnutzen. Die Anforderungen an den Technologieeinsatz kommen somit vom Kunden und anwendenden Mitarbeitenden und können nicht isoliert formuliert werden. Diese konsequente und frühzeitige Einnahme der Kundenperspektive muss auch für die Gestaltung, Ausführung und Weiterentwicklung der Prozesse (Prozessziele, -struktur, IT-Unterstützung) 
- Welchen Beitrag leistet das Prozessmanagement für die Maximierung des Kundennutzens durch:

- die Veränderung des Kundenerlebnisses?

- die Schaffung von Operational \& Service Excellence?

- die Innovation von Produkten und Dienstleistungen?

- Wird durch diesen Beitrag das Geschäftsmodell erneuert/erweitert?

- Entstehen neue digitale oder digital erweiterte Produkte und Dienstleistungen?

- Entstehen neue Partner-Plattformen/Netzwerke?

○ Werden Ertragskraft und Wettbewerbsposition gestärkt?

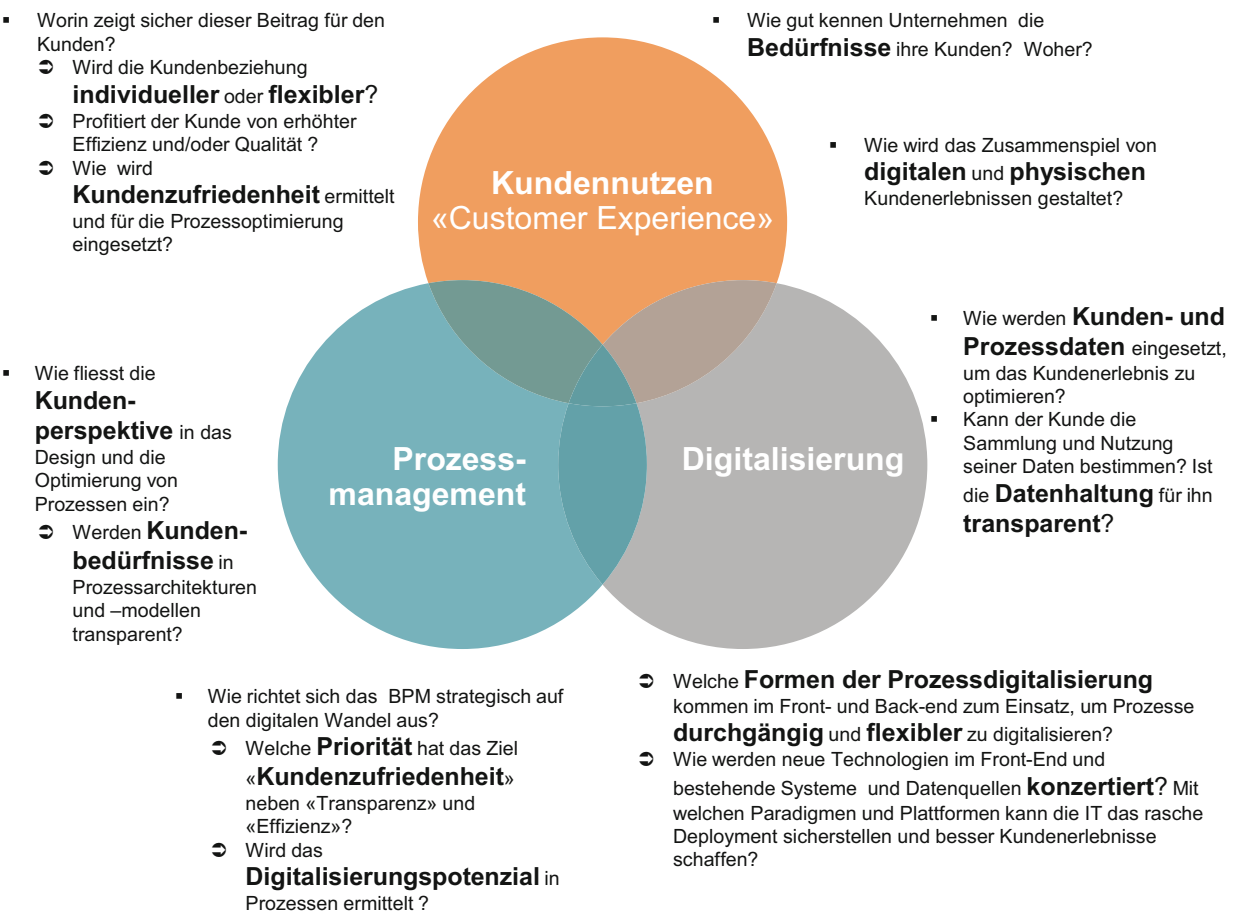

- Abb. 1.2 Forschungsfragen

gelten. Die konsequente Ausrichtung auf den Kundenbedarf wird im Prozessmanagement heute vor allem unter dem Prinzip «End-to-End-Prozessmanagement» zusammengefasst. Endto-End-Prozessmanagement konzentriert sich jedoch auf das Kundenbedürfnis im Sinne eines Prozessinputs und dessen Erfüllung im Sinne eines Prozessoutputs, der häufig mit dem Prozessziel gleichgesetzt wird. Der durchgängige Prozess selbst wird aus der unternehmensinternen Perspektive gestaltet, ohne den Berührungspunkten zum Kunden besondere Beachtung zu schenken. Für die Kundenbeziehung oder das Kundenerlebnis kritische Aktivitäten oder Informationsflüsse sind in Prozessmodellen typischerweise nicht speziell gekennzeichnet. Customer Journeys hingegen, die die Befindlichkeiten des Kunden an allen Berührungspunkten 
mit dem Unternehmen abbilden (Gartner Research 2014), werden isoliert im Marketing eingesetzt und haben häufig keinen Bezug zu den Prozessmodellen.

\section{- Prozessdigitalisierung: die richtige Form und das richtige Mass}

Digitalisierung wird in vielen Unternehmen heute ausschliesslich im Front-End für die Optimierung des Kundenerlebnisses oder ausschliesslich im Back-End für Standardisierung und Effizienzsteigerung betrieben. Diese isolierten Aktivitäten sind nicht zielführend. Durchgängige und flexible Prozesse, die Effizienz und Kundenerlebnis gleichermassen optimieren, werden nur durch Integration von Front- und Back-End und gegebenenfalls externen Partnern und Informationssystemen möglich. Insbesondere kleinen und mittleren Unternehmen fehlt häufig die Infrastruktur, um diese Integration rasch und wiederverwendbar zu realisieren. Diese «harte Realität» der digitalen Transformation wird mit Blick auf prestigeträchtige Technologien an der Schnittstelle zum Kunden (z. B. mobile Apps), gerne ausgeblendet. Auch das breite Spektrum der Prozessdigitalisierung wird häufig nicht ausgenutzt. Prozessdigitalisierung umfasst mehr als nur die Automatisierung von Standardprozessen. Die IT-Unterstützung schwach strukturierter, wissensintensiver Prozesse, ein wesentlicher Aspekt des digitalen Arbeitsplatzes, ist in Unternehmen immer noch schwach ausgeprägt. Methoden, um zielgerichtet Prozesse zu digitalisieren, das heisst, die passende Digitalisierungsform und den optimalen Grad an Standardisierung beziehungsweise Flexibilisierung oder Befähigung zu finden, existieren kaum. Auch die Frage, ob Technologieeinsatz Kundennutzen immer erhöht oder unter Umständen auch minimiert oder gar zerstört, wird in Zeiten hektischer Digitalisierungsinitiativen nicht systematisch adressiert.

\section{- Digitalisierung = datenzentrisches Prozessmanagement}

Daten sind der Treibstoff der digitalen Transformation und spielen auch für die kundenorientierte Ausgestaltung, Flexibilisierung und Optimierung der Prozesse eine zentrale Rolle. Die Digitalisierung der Kundenbeziehung führt zwangsläufig zu einem massiven Wachstum der Kundendaten. Damit verbunden sind nicht nur neue Möglichkeiten, Kundenerlebnisse auch in der digitalen Welt individuell zu gestalten oder innovative Produkte und Dienstleistungen anzubieten, sondern die Verantwortung der Unternehmen, über das gesetzliche Mindestmass hinaus Transparenz über die Haltung und Verwendung dieser Daten herzustellen. Unternehmen sind sich dieser Verantwortung bewusst und auf der Suche nach geeigneten Mechanismen, diese Transparenz zu gewährleisten und so eine vertrauensvolle Kundenbeziehung nachhaltig sicherzustellen.

\subsubsection{Vorgehen und Ergebnisse}

Die Studie umfasst einen quantitativen und qualitativen Forschungsteil:

- Quantitativ - Status quo in der Praxis: Im Rahmen einer Online-Befragung wird die Ausprägung, mit der Unternehmen im deutschsprachigen Raum Konzepte, Methoden und Technologien an den Schnittstellen von Prozessmanagement, Kundenorientierung und Digitalisierung einsetzen, erhoben.

- Qualitativ - Erfolgsmuster in Unternehmen: Im Rahmen offener Interviews und eines ganztägigen Workshops werden Fallstudien mit Unternehmen erarbeitet, die ihre Erfahrungen, Lösungsansätze und Resultate aus kundenzentrischen Digitalisierungs- bzw. Prozessmanagement-Initiativen aufzeigen. 


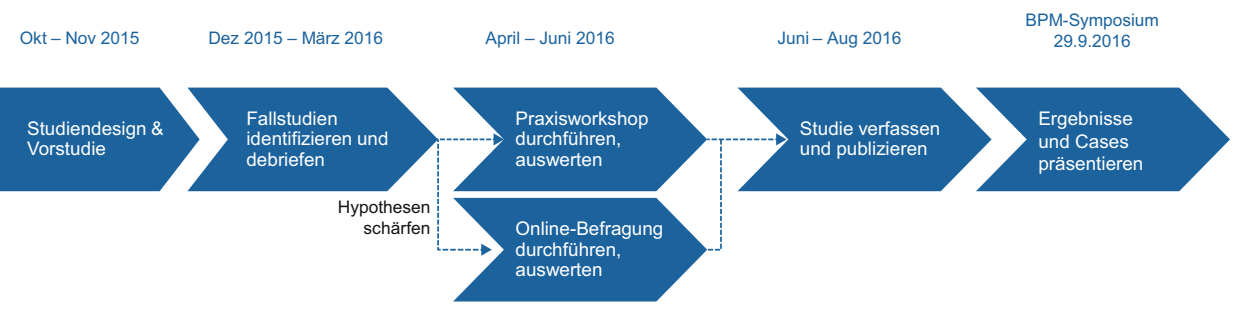

- Abb. 1.3 Studiendesign und Ablauf

Tab. 1.1 Elemente und Ergebnisse der Studie

\begin{tabular}{|c|c|c|}
\hline & Vorgehen & Ergebnisse ( $\rightarrow$ Kapitel) \\
\hline $\begin{array}{l}\text { Was ist digitale Transformati- } \\
\text { on? Welche Handlungsfelder } \\
\text { eröffnen sich für das Prozess- } \\
\text { management? }\end{array}$ & $\begin{array}{l}\text { Vorstudie: } \\
\text { - Definitionen und Gestaltungsebe- } \\
\text { nen der «digitalen Transformation» } \\
\text { - Stand der Forschung «BPM \& } \\
\text { digitale Transformation» }\end{array}$ & 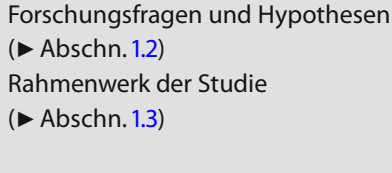 \\
\hline $\begin{array}{l}\text { In welcher Ausprägung setzen } \\
\text { Unternehmen Konzepte, Me- } \\
\text { thoden und Technologien an } \\
\text { den Schnittstellen zwischen } \\
\text { Prozessmanagement, Kunden- } \\
\text { nutzen und Digitalisierung } \\
\text { ein? }\end{array}$ & $\begin{array}{l}\text { Quantitativ: } \\
\text { - Online-Befragung von Unterneh- } \\
\text { men im deutschsprachigen Raum } \\
\text { (April-Mai 2016) }\end{array}$ & $\begin{array}{l}\text { Umfrage-Ergebnisse ( Kap. 2) } \\
\text { Fazit: Status quo ( } \$ \text { Abschn. 9.1) }\end{array}$ \\
\hline $\begin{array}{l}\text { Welche Erfahrungen, Lösungs- } \\
\text { ansätze und Resultate weisen } \\
\text { Unternehmen auf, die den } \\
\text { Kundennutzen und die Mög- } \\
\text { lichkeiten der Digitalisierung } \\
\text { ins Zentrum ihrer BPM-Initiati- } \\
\text { ven stellen? }\end{array}$ & $\begin{array}{l}\text { Qualitativ: } \\
\text { - Debriefings mit Fallstudienkandi- } \\
\text { daten } \\
\text { - Ganztägiger Praxis-Workshop mit } \\
5 \text { Unternehmen }\end{array}$ & $\begin{array}{l}\text { Fallstudien von Amag Leasing, } \\
\text { Vontobel, Kanton Zürich, Swiss Re } \\
\text { und Swisscom ( Kap. 4-8) } \\
\text { Fazit: Erfolgsmuster ( Abschn. 9.2) }\end{array}$ \\
\hline $\begin{array}{l}\text { Welche Konsequenzen } \\
\text { ergeben sich für das Prozess- } \\
\text { management? }\end{array}$ & & $\begin{array}{l}\text { Fazit: Quo vadis BPM? } \\
\text { ( } \triangleright \text { Abschn. 9.3) }\end{array}$ \\
\hline
\end{tabular}

- Abb. 1.3 skizziert das Vorgehen. - Tab. 1.1 fasst den Inhalt der vorbereitenden sowie quantitativen und qualitativen Forschungsteile und deren Ergebnisse zusammen.

\subsection{Das Rahmenwerk der Studie}

«Kundennutzen durch digitale Transformation?» lautet der Untertitel der Business-ProcessManagement-Studie 2016. Das Fragezeichen steht für eine Vielzahl offener Fragen und Zweifel, die sich bei der Realisierung dieses Heilsversprechens früher oder später einstellen. Die Vision des Endzustands kling verlockend: Kunden profitieren von innovativen Produkten und Dienstleistungen, die nicht nur effizienter erbracht werden, sondern eine neue Dimension von Kundenerlebnissen eröffnen. Unternehmen stehen allerdings vor der Aufgabe, die Voraussetzungen zu schaffen, das heisst nicht nur ihr traditionelles Geschäftsmodell, ihre Strategien und 


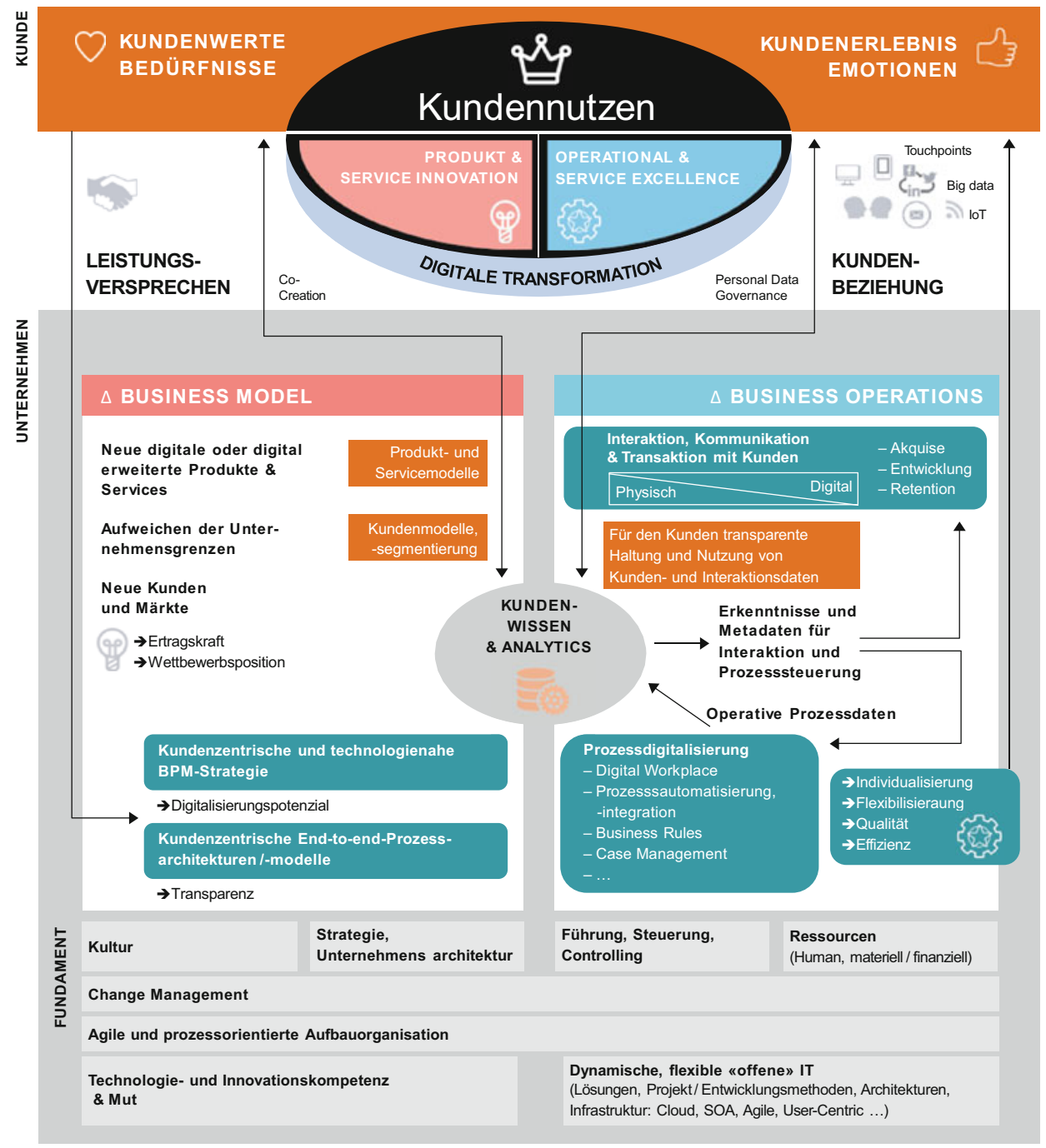

- Abb. 1.4 Rahmenwerk der BPM Studie: Kundennutzen durch digitale Transformation? Gestaltungsfelder und Potenziale für das Prozessmanagement

ihre Kultur zu hinterfragen, zu verändern oder zumindest anzupassen, sondern auch ihre betrieblichen Strukturen, Abläufe und Systeme so weit zu entwickeln, dass sie eine Öffnung in Richtung Kunde zulassen. Gestaltungsfelder sind also vorhanden. Doch wo und wie wirkt das Prozessmanagement?

Um mögliche Wirkungsbereiche des Prozessmanagements im Kontext der digitalen Transformation herauszuarbeiten, wurde im Rahmen der Vorstudie ein Rahmenwerk entworfen, das die Gestaltungsfelder der digitalen Transformation aufzeigt (- Abb. 1.4). Das Rahmenwerk diente zum einen als Arbeitsdefinition für den Begriff der digitalen Transformation und zum anderen als Projektionsfläche, um strategische, fachliche sowie technische Lösungselemente des Prozessmanagements in diesem Gestaltungsrahmen zu platzieren. Im qualitativen 
Forschungsteil wurde das Rahmenwerk eingesetzt, um die Fallstudien der beteiligten Unternehmen einzuordnen, Erfolgsfaktoren zu identifizieren und Entwicklungspotenziale zu diskutieren.

Das Rahmenwerk basiert auf bestehenden Definitionen ( Abschn. 1.1) und betrachtet die digitale Transformation aus drei Perspektiven:

1. Wozu und für wen wird digital transformiert?

- Kundennutzen

- Produkt- und Service-Innovation

- Operational und Service Excellence

2. Was wird digital transformiert?

- Kundenerlebnis

- Geschäftsmodell

- Business Operations

3. Wie beziehungsweise womit wird digital transformiert (Fokus: Prozessmanagement)?

- Strategisch, konzeptionell:

- Kundenzentrische und technologienahe BPM-Strategien: Digitalisierungspotenzial (und Digitalisierungsgrenzen) identifizieren

- Kundenzentrische End-to-End-Prozessarchitekturen und -modelle: Transparenz: Kundennutzen und für die Kundenbeziehung kritische Punkte sichtbar machen

- Operativ, technisch:

- Formen der Prozessdigitalisierung zweckmässig einsetzen für:

- Optimierung von Effizienz und/oder Qualität

- Flexibilisierung und Individualisierung von Kundeninteraktionen

- Synchronisierung von physischen und digitalen Kundenerlebnissen

- Kundenwissen \& Customer Analytics:

- Kundendaten und operativen Prozessdaten für die flexible Prozesssteuerung einsetzen (retrospektiv und in Echtzeit)

- Transparenz der Datenhaltung für Kunden sicherstellen

\subsubsection{Digitale Transformation - Wozu und für Wen?}

Was möchten Unternehmen durch digitale Transformation erreichen und wer profitiert von diesen Errungenschaften (- Abb. 1.5)? Wie bereits in $>$ Abschn. 1.1 ausgeführt, steht der Kunde mit seinen Bedürfnissen, Emotionen und persönlichen Werthaltungen im Fokus der digitalen Transformation. Technologien einzusetzen, um positive Kundenerlebnisse zu schaffen, ist eine nachvollziehbare Motivation. Die Realisierung dieser Erlebnisse und der effektive Wirkungsnachweis fallen jedoch schwer. Kundenbedürfnisse sind häufig nicht ausgesprochen und werden von Unternehmen lediglich angenommen und in Anforderungen formuliert, sei es für die Entwicklung eines Produkts oder einer Dienstleistung oder für den Entwurf eines Prozesses oder einer Softwarelösung. Der Kundennutzen wird im Customer Experience Management als relativer Wert betrachtet («perceived value»), der je nach Situation und Erfahrungshintergrund von jedem Kunden individuell empfunden wird. Objektive, auch vom Prozessmanagement traditionell anvisierte Grössen wie Schnelligkeit, Qualität oder Preis werden so zu subjektiven Grössen, die den Kundennutzen zweifelsohne beeinflussen, jedoch nicht für jeden Kunden und in jedem Kontext gleichermassen relevant sind.

Dies hat auch Einfluss auf die kontinuierliche Entwicklung der Operational und Service Excellence. Die Optimierung von Prozessen im Back- und Front-End hinsichtlich Qualität 


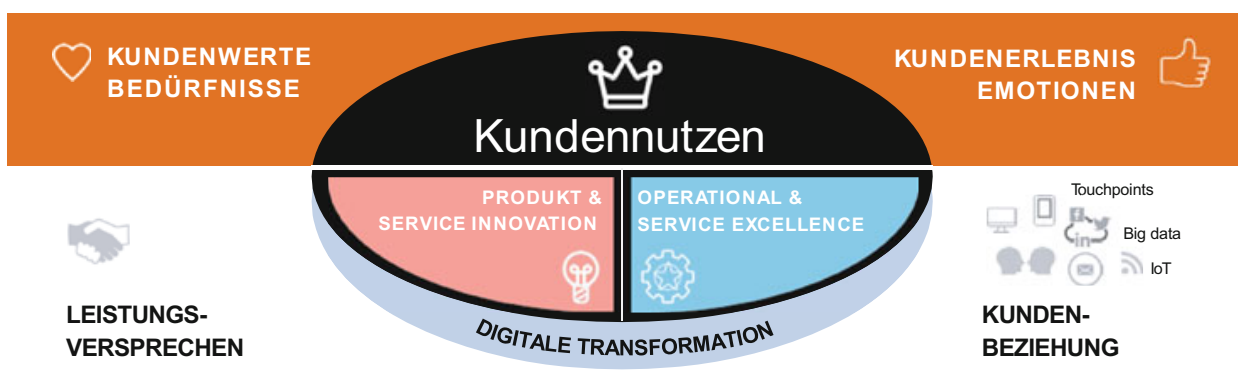

- Abb. 1.5 Gestaltungsziele der digitale Transformation - Wozu und für Wen?

und Effizienz stellt eine weitere wesentliche Motivation für die digitale Transformation in Unternehmen dar. Vor allem an der Schnittstelle zum Kunden, aber auch zu Partnern und einer neuen Generation von Mitarbeitenden, ist jedoch nicht immer fassbar, welche Leistung unter welchen Bedingungen als exzellent betrachtet wird. Flexibilität und Reaktionsfähigkeit, in der Regel in Echtzeit, sind eine neue Dimension von Operational und Service Excellence, die hohe Anforderungen an Daten und Prozessintegration mit sich bringt.

Die Innovation von Produkten und Services ist Motivation und Treiber der digitalen Transformation zugleich. Technologische Neuerungen sind die wesentliche Kraft hinter vielen Produkt- und Service-Innovationen in den verschiedensten Branchen. Neue Wettbewerber nutzen diese Chance, um mit innovativen digitalen Dienstleistungen in den Markt zu drängen. Aber auch etablierte Anbieter erweitern ihr Produkt- und Dienstleistungsportfolio um digitale Innovationen. Grundvoraussetzung ist ein offenes Innovationsmanagement, das neue Konzepte wie Design Thinking in interdisziplinären, unternehmensweiten und -übergreifenden Teams und die Einbindung von Kunden in Produktentwicklungs- und Innovationsprozesse («CoCreation») aufgreift.

\subsubsection{Digitale Transformation - Was wird transformiert?}

Reichweite und Wirkungsfelder der digitalen Transformation innerhalb des Unternehmens und für den Kunden können unterschiedlich ausgeprägt sein: von der Ergänzung oder Optimierung eines Online-Kanals, über die Ausstattung von Aussendienstmitarbeitern mit mobilen Applikationen oder der durchgängigen Automatisierung eines Prozesses bis hin zu einer Veränderung oder Erweiterung des Geschäftsmodells. Grosses Potenzial liegt vor allem in den Wirkungszusammenhängen zwischen den Transformationsbereichen Kundenerlebnis, Geschäftsmodell und Business Operations (-Abb. 1.6). Werden Produkte, Dienstleistungen oder Distributionskanäle digital erweitert, muss sich dies auch in den Prozessarchitekturen und -modellen niederschlagen, die wiederum einen Beitrag leisten, neue und veränderte Prozesse durchgängig und konsequent am Kundenbedarf ausgerichtet zu entwerfen. Voraussetzung ist eine enge Verzahnung der Prozessarchitektur und -modelle mit der Geschäfts- und Informationssystemarchitektur (z. B. durch die Hinterlegung von Geschäftsobjekten, Applikationen, Datenquellen), um Integrationsherausforderungen, Informationsbedürfnisse und Digitalisierungspotenziale oder -defizite offenbar werden zu lassen. Im Idealfall spielt ein derart kundenzentrisch und technologienah aufgestelltes Prozessmanagement eine aktive Rolle in der Identifikation von Business Cases für die die Prozessdigitalisierung im Front- und Back-End. 


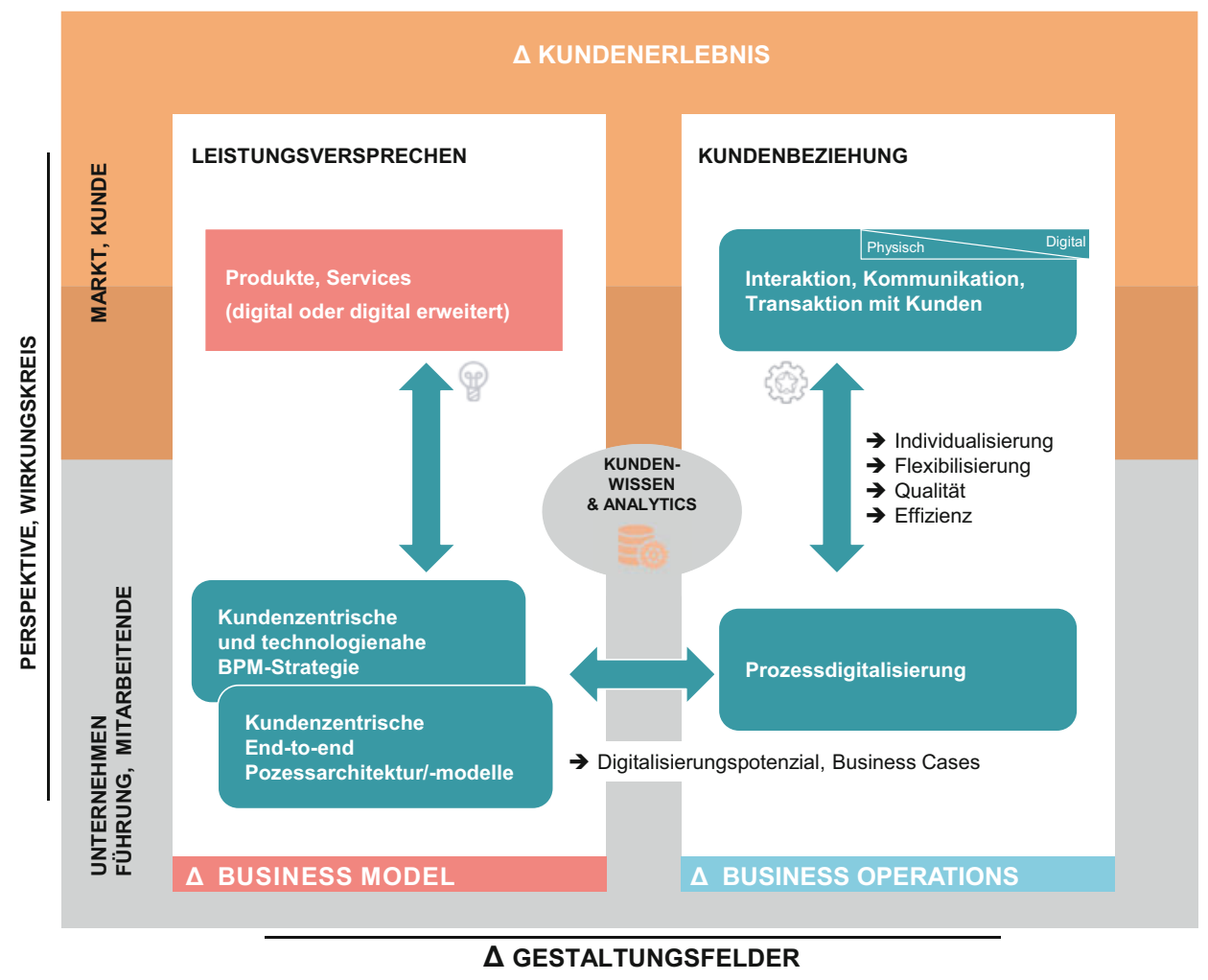

- Abb. 1.6 Gestaltungsfelder der Digitalen Transformation - Was wird transformiert?

\subsubsection{Digitale Transformation - Womit? (Fokus: Formen der Prozessdigitalisierung)}

Digitalisierung wird häufig mit technologischen Gadgets, Sozialen Netzwerken und mobilen Applikationen assoziiert, die in erster Linie Kunden neue Interaktionsmöglichkeiten mit dem Unternehmen und dessen Produkten und Dienstleistungen eröffnen. Prozessdigitalisierung rückt erst beim Stichwort «Operational Excellence» ins Bild und wird typischerweise mit der Automatisierung stark strukturierter und standardisierter Prozesse gleichgesetzt. Doch diese enge Sicht deckt nur einen Teil des Spektrums ab. Die Schaffung herausragender Kundenerlebnisse im Front-End und durchgängiger Prozesse setzt eine sehr viel breitere Technologiebasis in Unternehmen voraus (- Abb. 1.7), die teilweise bereits vorhanden, aber nicht immer integriert ist und nicht immer auf den neuesten Technologien oder Systemarchitekturen beruht. Im Rahmen der vorliegenden Studie werden unter dem Begriff Prozessdigitalisierung insbesondere auch der Technologieeinsatz für schwach strukturierte und wissensintensive Prozesse verstanden, die die Kollaboration und Entscheidungsunterstützung von Mitarbeitenden, gegebenenfalls mit Einbezug von Kunden oder Partnern, unterstützen. 


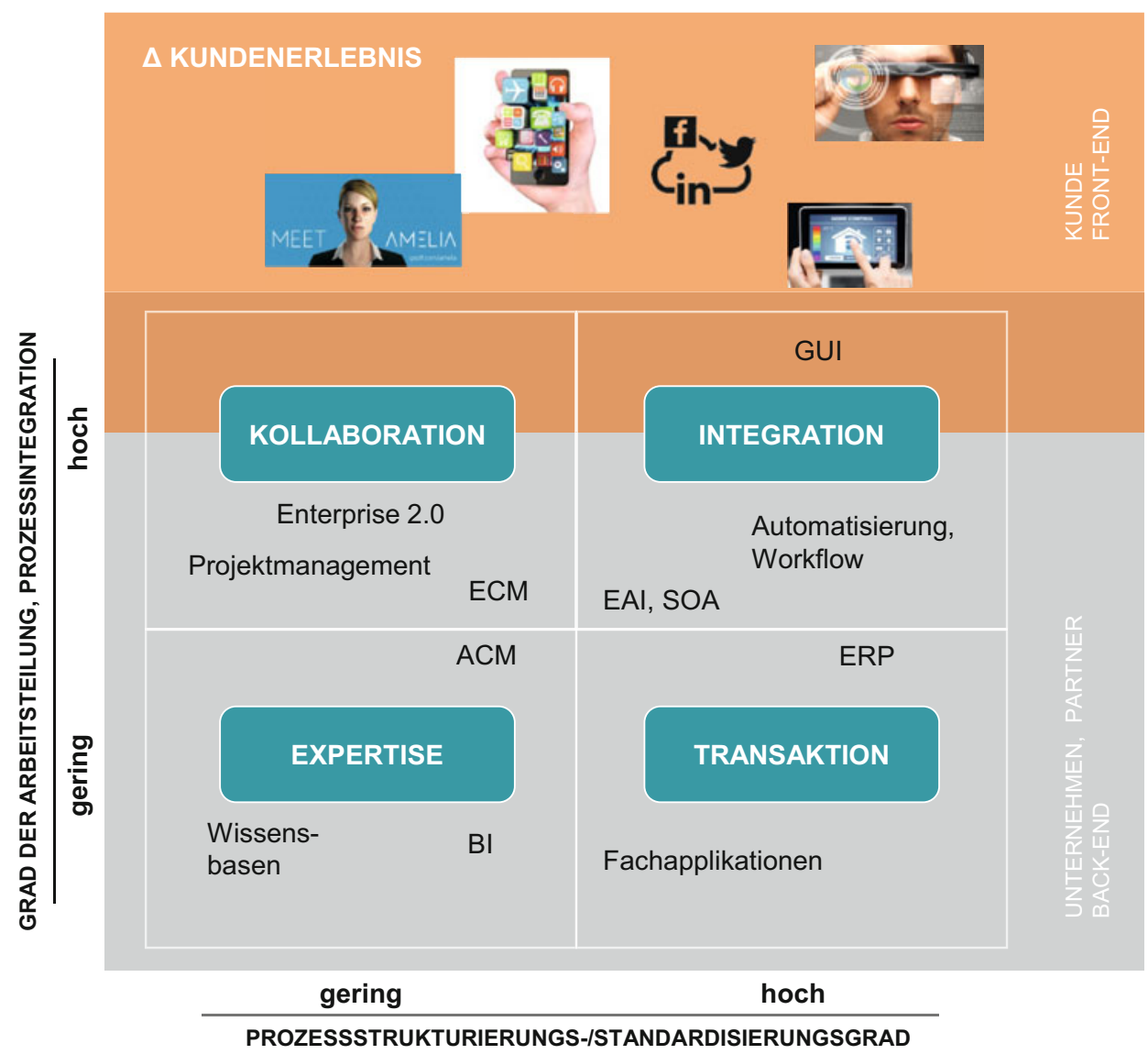

- Abb. 1.7 Digitale Transformation - Wie und Womit? (Formen der Prozessdigitalisierung)

\section{Literatur}

BPM\&O, \& BearingPoint (2015). Business Process Management Studie 2015 - Messbare Verbesserung der Leistungsfähigkeit durch Prozessmanagement. Frankfurt am Main: BearingPoint.

Brucker-Kley, E., Kykalová, D., Grünert, D., Keller, T., Schertenleib, R., Schlatter, U., \& Schwer, K. (2015). Business Process Management 2015: Status quo und Best Practices »Prozessintelligenz" - Eine Studie des Instituts für

Wirtschaftsinformatik. Winterthur: ZHAW School of Management and Law.

Crisp Research (2015). Digital Leader - Leadership im digitalen Zeitalter. Kassel: Crisp Research.

Fitzgerald, M., Kruschwitz, N., Bonnet, D., \& Welch, M. (2014). Embracing digital technology: a new strategic imperative. MIT sloan management review, 55(2), 1-12.

Frick, K., \& Höchli, B. (2014). Die Zukunft der vernetzten Gesellschaft. Rüschlikon, Zürich: GDI.

Gartner Research (2014). Use journey maps in user experience design. Stamford: Gartner.

McDougall, G., \& Levesque, T. (2000). Customer satisfaction with services: putting perceived value into the equation. Journal of services marketing, 14(5), 392-410.

McKinsey \& Co (2015). What digital really means. http://www.mckinsey.com/insights/high_tech_telecoms_ internet/what_digital_really_means. Abgerufen am 26.06.2017

Pine, B. J., \& Gilmore, J. H. (1998). Welcome to the experience economy. Harvard Business Review, 76, 97-105. 
Stolterman, E., \& Fors, A. C. (2004). Informationtechnology and the good life. Information Systems Research, 143, 687-692.

Universität St.Gallen; Crosswalk (2016). Digital maturity \& transformation report. St.Gallen: Institut für Wirtschaftsinformatik, Universität St.Gallen.

Open Access Dieses Kapitel wird unter der Creative Commons Namensnennung 4.0 International Lizenz (http://creativecommons.org/licenses/by/4.0/deed.de) veröffentlicht, welche die Nutzung, Vervielfältigung, Bearbeitung, Verbreitung und Wiedergabe in jeglichem Medium und Format erlaubt, sofern Sie den/die ursprünglichen Autor(en) und die Quelle ordnungsgemäß nennen, einen Link zur Creative Commons Lizenz beifügen und angeben, ob Änderungen vorgenommen wurden.

Die in diesem Kapitel enthaltenen Bilder und sonstiges Drittmaterial unterliegen ebenfalls der genannten Creative Commons Lizenz, sofern sich aus der Abbildungslegende nichts anderes ergibt. Sofern das betreffende Material nicht unter der genannten Creative Commons Lizenz steht und die betreffende Handlung nicht nach gesetzlichen Vorschriften erlaubt ist, ist für die oben aufgeführten Weiterverwendungen des Materials die Einwilligung des jeweiligen Rechteinhabers einzuholen.

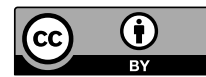

